\section{Local anaesthesia for vitreoretinal surgery: a case-control study of 200 cases}
Abstract
Purpose In the United Kingdom the majority of vitreoretinal (VR) surgery is performed under general anaesthesia (GA). The aim of this study was to demonstrate the scope of local anaesthesia (LA) for VR surgery, to measure the acceptance of LA to patients and surgeons and to compare the surgical outcomes, complication rates and duration of the surgical procedures under LA and GA. Methods A case-control study was undertaken to compare 100 cases performed under LA with 100 matched cases performed under GA. The matching of cases was based on multiple criteria such as configuration and complexity of retinal detachment, the involvement of the macula, the number and site of retinal tears, presence and severity of proliferative vitreoretinopathy, experience of the surgeon and the type of the surgical procedure. A clinical audit was also carried out on 65 successive patients using a questionnaire to determine the acceptability of LA to patients and surgeons.
Results Anatomical and visual success rates, and intra-operative and post-operative complications, were similar in cases carried out under LA and GA. The mean duration of the surgery (excluding anaesthetic time) was
the time of operation. Manoeuvres such as cryotherapy, scleral buckling and traction on the globe are often painful and delicate dissection close to the retina requires total akinesia. A straw poll conducted at the BEAVRS (British and Eire Association of Vitreoretinal Surgeons) meeting indicated that the majority of VR surgery in the UK is carried out under general anaesthesia (GA). ${ }^{3}$
Until recently, virtually all the VR surgery in our unit was carried out under GA. In 1995 we made a conscious decision to employ LA. The decision was prompted by the recent favourable reports indicating the feasibility of VR surgery with LA and its high patient acceptance. ${ }^{4-6}$ In 1994 in St Paul's Eye Unit, just over 1\% (5 of 405 cases) of VR surgery was carried out under LA and in 1995 this proportion had increased to $35 \%$ (183 of 510 cases). This abrupt change in clinical practice gave us an opportunity and an onus to audit its effect. We therefore conducted this case-control study with the following aims:
1. to demonstrate the scope of LA,
2. to compare the surgical outcomes and complications of LA versus GA,
3. to measure the duration of surgery under LA and GA,
4. to measure the acceptance of LA to patients undergoing VR surgery. significantly shorter for LA than GA procedures $(p<0.001)$. The acceptance for LA was high for both patients and the operating surgeons.

Conclusions We found that VR surgery can be safely and efficiently performed under LA.

Adoption of LA has increased our throughput.

Key words Local anaesthesia, Retinal detachment, Surgery, Vitreoretinal

Local anaesthesia (LA) for ocular surgery in the form of a retrobulbar injection was first described by Knapp in $1884 .{ }^{1}$ In recent years, LA has been adopted widely for cataract operations. ${ }^{2}$ Vitreoretinal (VR) surgery, unlike cataract extraction, tends to be prolonged and less predictable. The surgical steps occasionally need to be modified according to the findings at
G. PRASAD RAO, DAVID WONG, CARL GROENEWALD, JAMES N. MCGALLIARD, ANDREW JONES, PETER J.G. RIDGES

\section{Methods}

The data base for the study was 405 VR procedures carried out in 1994 and 510 operations carried out in 1995. The type of anaesthesia used is given in Table 1 . We compared cases carried out under LA in 1995 with matched cases carried out under GA in 1994.

\section{Matching}

To avoid selection bias, we matched each case under LA with another case under GA using multiple criteria. The case matching was performed by one of us (G.P.R.) who was not involved with any of the surgery. The criteria we used were those that we had found from a previous prospective, pan-regional audit to be
G.P. Rao

D. Wong

C. Groenwald

J.N. McGalliard

St Paul's Eye Unit

Royal Liverpool University

Hospital

Prescot Street

Liverpool L7 8XP, UK

Tel: $+44(0) 1517062000$

Fax: +44 (0)151706 5861

A. Jones

Department of Anaesthesia Royal Liverpool University

Hospital

Liverpool, UK

P.J.G. Ridges

Department of Orthoptics The University of Liverpool Liverpool, UK 
Table 1. Vitreoretinal activity 1994 versus 1995

\begin{tabular}{lcc}
\hline & 1994 & 1995 \\
\hline LA (no. of patients) & 5 & 183 \\
GA (no. of patients) & 400 & 327 \\
Total (no. of patients) & 405 & 510 \\
\hline
\end{tabular}

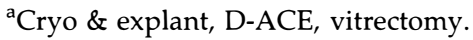

influential to the outcome of VR surgery. ${ }^{7}$ These criteria (Table 2) included the configuration of rhegmatogenous retinal detachment (RRD); the number, type and site of retinal breaks; the numbers of quadrants of detached retina; the involvement of macula in the $\mathrm{RD}$; the status of the lens; the presence and severity of proliferative vitreoretinopathy (PVR); the type of surgical procedure used and the operating surgeon. In this way we avoided the possibility that LA was being applied to the 'simpler' cases of RRD. There were VR procedures carried out for indications other than for RRD (Table 2) that were also matched for degree of complexity in order to make a fair and reasonable comparison. We were able to match 100 of the 183 procedures carried out under LA in 1995 with 100 of 400 carried out under GA in 1994. Information was collected using a standard proforma. Pre-operative and post-operative visual acuities at 1 month and 3 months were noted.

\section{Anatomical success}

In cases of RRD, anatomical success was defined as total retinal re-attachment at 3 months post-operatively.

\section{Visual success}

We defined visual improvement as a gain of at least 1 Snellen line or more over the pre-operative visual acuity at the 3 months post-operative visit.

Table 2. Matching of cases under local anaesthesia ( $L A)$ and general anaesthesia (GA)

\begin{tabular}{lrr}
\hline Criteria & LA & GA \\
\hline Bullous detachment & 16 & 18 \\
Multiple tears & 22 & 24 \\
Total detachment & 14 & 12 \\
Pseudophakia & 13 & 11 \\
Re-do & 5 & 6 \\
Dialysis & 3 & 2 \\
PVR & 6 & 6 \\
Diabetic eye disease & 16 & 18 \\
Dropped nucleus & 4 & 3 \\
Trauma & 2 & 2 \\
Macular holes & 3 & 2 \\
Macular pucker & 2 & 4 \\
SRNVM & 1 & 1 \\
Vitreous haemorrhage due to PVD/RB & 3 & 2 \\
Vitreous haemorrhage due to C/BRVO & 4 & 4 \\
Endophthalmitis & 1 & 1 \\
\hline
\end{tabular}

Re-do, repeat operations; PVR, proliferative vitreoretinopathy; SRNVM, subretinal neovascular membrane; PVD, posterior vitreous detachment; $\mathrm{RB}$, retinal break; $\mathrm{C} / \mathrm{BRVO}$, central/ branch retinal vein occlusion.

\section{Duration of surgery}

As part of our routine theatre data collection, the duration of surgery and the name of the principal surgeon were recorded by the nursing staff, who were unaware of the study. The duration of surgery did not include time for anaesthesia, which was recorded separately.

\section{Method of local anaesthesia}

We applied a single transconjunctival anterior retrobulbar injection in the inferotemporal quadrant using $2 \%$ lignocaine with 1500 units of hyalase and a 1 inch 25 gauge needle. Six millilitres of the anaesthetic solution was injected. Top-up injections were used for operations lasting more than $1 \mathrm{~h}$.

\section{Audit of the acceptability of LA}

A clinical audit was carried out between November 1995 and February 1996 in the VR unit, of 65 successive patients using a questionnaire. Patients were asked two questions:

1. Would you undergo VR surgery under LA again?

2. Was the operation painful?

These questions were asked immediately after the operation whilst the patient was in the recovery suite in the theatre. Patients were asked to grade the intraoperative discomfort on a 10-point visual analogue score.

The operating surgeon was asked:

1. Would you undertake the operation under LA again?

2. Were there any complications that have arisen as a result of or related to LA?

\section{Statistical analysis}

The difference in mean duration of surgical procedure for the two groups was calculated, and the associated confidence intervals calculated using the method of a paired $t$-test. To compare the numbers of successes and complications, odds ratios were calculated. Confidence intervals for odds ratios were calculated using logit limits, except where numbers were very small, when Cornfield's limits were calculated.

\section{Results}

\section{Scope of LA}

This series has shown that it is possible to carry out VR surgery under LA for a wide range of indications (Table 2).

\section{Matching}

Thirty-one patients underwent the $\mathrm{D}-\mathrm{ACE}^{8}$ surgical sequence and 19 patients received cryotherapy and scleral buckling under LA and under GA for the repair of 
Table 3. Matching by procedures and surgeons

\begin{tabular}{lll}
\hline & LA & GA \\
\hline D-ACE & 31 & 31 \\
Cryo \& explant & 19 & 19 \\
Vitrectomy & 50 & 50 \\
Consultant & 45 & 59 \\
Trainees & 55 & 41 \\
\hline
\end{tabular}

D-ACE, drainage, air, cryotherapy and explant.

RRD. A further 50 subjects had vitrectomy procedures under LA and the same number of patients under GA (Table 3). The indications for vitrectomy were principally the repair of RRDs and involved scleral buckling in most cases. Other indications for vitrectomy include diabetic eye disease, dropped nucleus, posterior segment trauma, macular hole, macular pucker, vitreous haemorrhage due to venous occlusive disease, subretinal neovascular membrane removal and endophthalmitis. There were similar number of patients undergoing LA and GA for each of these indications for surgery (Table 2). The matching according to the features of the RRD was good and there was no significant difference between LA and GA patients in terms of the bullous configuration,

multiplicity of breaks, type of breaks, phakic status and the presence of PVR. The numbers of operations carried out under LA and GA by consultants and trainees were similar (Table 3).

\section{Anatomical success}

Sixty of 68 (88\%) cases of RRD were successfully repaired under LA and 57 of $68(84 \%)$ cases under GA. There was no statistically significant difference between the two groups (odds ratio of 1.45 with $95 \%$ confidence interval of 0.54 to 3.86 ).

\section{Visual improvement}

Visual improvement of one or more Snellen lines occurred in 79 of 100 (79\%) cases undergoing surgery under LA and 80 of $100(80 \%)$ cases under GA. There was no significant difference between LA and GA (odds ratio of 0.94 with $95 \%$ confidence interval of 0.47 to 1.87 ).

\section{Complications (Table 4)}

Intra-operative complications under LA included haemorrhage from the drainage site in 4 patients, accidental globe perforation from deep scleral suture in 1 patient and iatrogenic retinal tear in another patient. Intra-operative complications under GA included one patient who had a drain site haemorrhage and another who suffered an iatrogenic tear during the peel of an adherent fibrovascular membrane. Post-operative complications occurred in 14 LA patients and $21 \mathrm{GA}$ patients. The most common post-operative complication was unresolved RD, and this was seen in 8 of the LA group compared with 12 of the GA group. We had no re-detachments in the LA group but there were 4 cases in the GA group. We encountered 2 cases of angle closure glaucoma in the GA group and 1 in the LA group.

\section{Duration of surgery (Table 4)}

The mean duration of surgery under LA was $57.02 \mathrm{~min}$ and under GA was $73.62 \mathrm{~min}$. The difference of $16.60 \mathrm{~min}$ was significant $(p<0.001)$ (the $95 \%$ confidence interval was 11.46 to 21.74$)$.

\section{Audit of $L A$}

Sixty of $65(92.3 \%)$ patients replied that they would undergo surgery under LA again. None of the 5 patients who would not have the surgery under LA again had discomfort scores greater than 6 . The surgeons replied that they would undertake the surgery again under LA in all 65 operations (100\%). There were no complications related to the LA. The visual analogue pain score result is shown in Fig. 1. Fifty-one of 65 patients $(78 \%)$ had pain

Table 4. Outcomes, duration and complications

\begin{tabular}{|c|c|c|c|c|}
\hline & LA & GA & Odds 1 & atio ( $95 \%$ confidence interval) \\
\hline \multicolumn{5}{|l|}{ Anatomical success } \\
\hline All cases & 90 & 88 & & $1.23(0.50$ to 2.99$)$ \\
\hline Rhegmatogenous RDs only & 60 & 57 & & $1.45(0.54$ to 3.86$)$ \\
\hline Visual success & 79 & 80 & & $0.94(0.47$ to 1.87$)$ \\
\hline Mean duration $(\min )$ & 57.02 & 73.62 & Difference & $16.60(11.46$ to 21.74$)$ \\
\hline Per-operative complications & (6) & (2) & & $0.32(0.07$ to 1.43$)$ \\
\hline Haemorrhage from drain site & 4 & 1 & & $0.24(0.04$ to 1.64$)$ \\
\hline Accidental perforation & 1 & 0 & & \\
\hline Iatrogenic tears & 1 & 1 & & \\
\hline Post-operative complications & (14) & (21) & & 1.63 (0.73 to 3.43$)$ \\
\hline Unsettled RD & 8 & 12 & & 1.57 (0.61 to 4.02$)$ \\
\hline Re-detachments & 0 & 4 & & (0.94 to infinity) \\
\hline Secondary glaucoma & 1 & 2 & & \\
\hline Others & $5^{\mathrm{a}}$ & $3^{\mathrm{b}}$ & & $0.59(0.15$ to 2.30$)$ \\
\hline
\end{tabular}

${ }^{a}$ VR incarceration, 1 ; endophthalmitis, 1 ; severe post-operative pain due to tight encirclement, 1 ; macular pucker, 1 ; secondary macular hole, 1.

${ }^{\mathrm{b}}$ Macular pucker, 3 . 


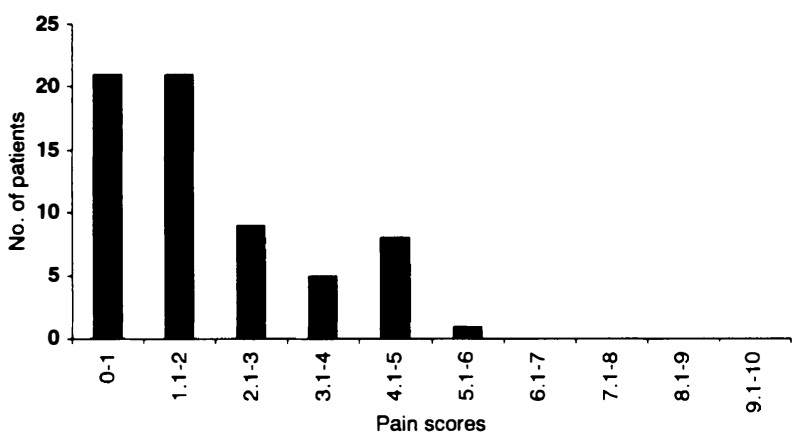

Fig. 1. Visual analogue pain scores of 6.5 LA patients.

scores of 3 or less. An anaesthetist was called to attend 2 patients; one had a self-limiting claustrophobic attack and the other required supplementary LA and sedation.

\section{Discussion}

In the USA, $85-90 \%$ of retinal re-attachment procedures are performed under LA, with GA generally reserved for complicated re-operations, detachments with breaks near the posterior pole, patients under the age of 20 years and those who have recently undergone anterior segment surgery, ${ }^{9,10}$ whereas in the United Kingdom the majority of all VR surgery is performed under GA. ${ }^{3} \mathrm{GA}$ is often a limited resource, not only in VR surgery but in many branches of surgery, and LA offers the attraction of possible faster turnover and increased efficiency. In common with many units in this country, there has been a steady increase in the workload of the VR unit in Liverpool. With increasing workload there is an everincreasing need for more efficient methods. We have been impressed by recent publications from the United Kingdom that have shown LA, without the adjunct of systemic sedation, to be effective and acceptable to patients and surgeons for VR surgery.

LA has some possible advantages over GA. It probably has lower cardiopulmonary complications, reduced incidence of post-operative nausea and vomiting and is suitable for patients who are at risk for general anaesthesia, such as those with sickle cell disease and uncontrolled diabetes. Patients do not need to be starved (usually for $6 \mathrm{~h}$ ) and can be taken to operating theatre directly, and this flexibility means that more cases can be performed within normal working hours. Patients who received LA are often able to commence posturing immediately if required to do so.

LA appears to be well tolerated by the patients; $92 \%$ of patients who we asked were willing to undergo the surgery again under LA. The experience of the surgeons has also been a positive one and in $100 \%$ of cases they expressed a readiness to undertake the surgery again using LA. The pain score was low, although most patients did report slight pain. The patients audited are selected as being willing to undergo surgery under LA. There were patients who were unwilling to receive operations under LA due to anxiety or other reasons.
Their views had not been sought. Unwillingness or anxiety is a limiting factor in the number of cases that can be performed under LA.

The duration of surgical procedures under LA ranged from $20 \mathrm{~min}$ to $130 \mathrm{~min}$. The effect of the initial local anaesthetic lasted on average up to an hour and any procedure that extend beyond $1 \mathrm{~h}$ required a top-up injection. We have shown that it is possible to perform VR surgery for a wide variety of indications under LA, including those that require lengthy and complex manoeuvres. It was possible to carry out macular surgery that involved delicate dissection close to the neurosensory retina, and we did not find poor akinesia to be a problem. Absolute akinesia was not necessary for most surgical manoeuvres.

In this study the anatomical re-attachment rates of VR procedures performed on RRDs were similar under LA and GA (88\% and $84 \%$ respectively). The visual improvement of more than one Snellen line was also similar between LA and GA (79\% and $80 \%$ respectively). These findings confirm the observations of Wilson and Barr. ${ }^{11}$ We are aware that the definition of visual improvement is an arbitrary one. The patients had a variety of pathology, including macular degeneration, diabetic retinopathy and venous occlusive diseases. In the group of patients with RRD, visual improvement depended on many factors such as the duration of RRD, the involvement of macula in the detachment and epimacular membranes. Nonetheless, we did not identify any evidence to suggest that the type of anaesthesia had a direct influence on achieving the desired surgical objectives and visual outcomes.

The overall complication rates were comparable between LA and GA and the difference was not statistically significant. The post-operative complication rates were higher in the GA group, though the difference was again not statistically significant (14 and 21; odds ratio 1.63 ).

The operative times were consistently shorter under LA compared with GA for similar procedures, and this difference was statistically significant (mean difference $16.60 \mathrm{~min} ; p<0.001$ ). We surmise that this shorter duration might be due to the fact that surgery under LA might be under time pressure and therefore the surgeon was more directed and purposeful. As a direct result of reduced operating time (and possibly anaesthetic time) under LA compared with GA it was in general possible to perform an extra case per theatre session. This allowed us to carry out more operations in 1995 than in 1994.

In summary VR surgery can be successfully carried out under LA as well as GA. We found that there was no difference between LA and GA in terms of anatomical and visual outcomes and intra-operative and postoperative complication rates. However, operative times were significantly shorter under LA compared with GA for similar procedures. VR surgery can be safely and efficiently performed under LA in selected patients. Adoption of LA has the potential of an increased throughput. 


\section{References}

1. Knapp H. On cocaine and its use in ophthalmic and general surgery. Arch Ophthalmol 1884;13:402-48.

2. Rosen E. Anaesthesia for ophthalmic surgery.

Br J Ophthalmol 1993;77:542-3.

3. Chignell AH. British and Eire Association of Vitreoretinal Surgeons' meeting, 1995, Loch Lomond (personal communication).

4. Mein CE, Woodcock MG. Local anaesthesia for vitreoretinal surgery. Retina 1990;10:47-9.

5. Stevens JD, Franks WA, Orr G, Leaver PK, Cooling RJ. Four quadrant local anaesthesia technique for vitreoretinal surgery. Eye 1992;6:583-6.

6. Arora R, Verma L, Kumar A, Tewari HK, Khosla PK. Peribulbar anaesthesia in retinal reattachment surgery. Ophthalmic Surg 1992;23:499-501.
7. McGailliard JN, Wong D. Results of a pan-regional prospective audit of primary retinal re-attachment surgery. Presented at BEAVRS meeting, 1993.

8. Gilbert C, McLeod D. D-ACE surgical sequence for selected bullous retinal detachments. Br J Ophthalmol 1985;69:733-6.

9. Ramsay RC, Knobloch WH. Ocular perforation following retrobulbar anaesthesia for retinal detachment surgery. Am J Ophthalmol 1978;86:61-4.

10. Isernhagen RD, Michels RG, Glaser BM, Bustros S, Enger C. Hospitalisation requirements after vitreoretinal surgery. Arch Ophthalmol 1988;106:767-70.

11. Wilson D, Barr CC. Outpatient and abbreviated hospitalisation for vitreoretinal surgery. Ophthalmic Surg 1992;21:119-22. 